\title{
Real-time estimation of free spaces in regulated on-street parking spaces using artificial neural networks
}

\author{
Miguel Magaña Suárez \\ Business Development Director, AUSSA, Spain \\ Miguel Núñez Timermans \\ Higher Technical School of Engineering, University of Seville, Spain
}

\section{SUMMARY}

In this paper we will develop a methodology for estimating the percentage of free parking spaces available in the area of the city where a user is interested through a real-time query in a mobile app. The smartphone screen will provide a colour-coded map of the requested area that indicates the saturation state of the parking spaces.

\section{INTRODUCTION}

The purpose of regulating on-street parking in cities is, among other things, to facilitate parking and reduce traffic issues in urban areas where deemed necessary by each regulatory administration. Regulated areas normally involve vehicles parked by residents and vehicles parking there temporarily, being managed through a rate that the user pays at enabled parking meters or through a mobile app.

The city being studied in this paper, Seville, consists of 7 large zones, each divided into sections or streets to facilitate their management. Estimates are made on a group of sections, hereinafter referred to as "area".

\section{AVAILABLE DATA}

Several real-time and historical data sources are available for use to estimate the real-time occupancy of parking spaces on street:

\subsection{Data derived from parking meters}

This source of data comes from tickets issued in real time at parking meters located on streets. This makes it possible to know the type of ticket bought by the user (visitor, resident, etc.), the interval of time reserved, the time of payment, and the parking meter where the operation takes place.

\subsection{Data derived from the mobile payment app}

Drivers use the PrestoParking app on the web or their smartphones to buy valid tickets to park in the regulated area. This information is accessible, though it is incomplete as it does not record the area of the city ultimately used by the user to park. 


\subsection{Data derived from counts}

Reports are collected from inspections carried out by employees/controllers related to vehicles parked in breach of ordinances. This inspection process is carried out systematically by traversing the parking areas and verifying the status of each space. The various situations that could arise are listed below:

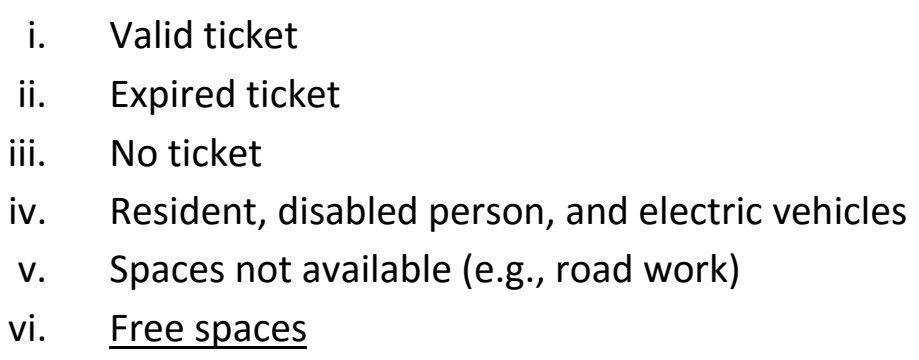

The available data are incomplete, as there is no exhaustive and complete real-time information on all of the sections or streets at all times, but rather discrete information for certain time intervals associated with the time periods during which the inspections are made.

\subsection{Weather data}

This type of data is a relevant source of information, as user behaviour is different on rainy days versus sunny days.

Historical data

The databases cited in the previous sections are filled in with the available records on the historical data of the same nature.

\section{MODELLING}

In this section, we present the methodological bases developed to predict the percentage of free spaces in a short time frame (30 minutes) in a certain area. An inverse relationship has been shown between the number of tickets sold in a certain area and the number of free spaces. However, it is not possible to make a sufficiently reliable estimate with only this set of information.

It should be noted that if the variable chosen as the most explanatory for the estimation is the number of tickets sold, it is necessary to have an estimate of that variable for the next time interval. This is why an artificial neural network (ANN) has been built for each of the existing parking meters in the parking area.

Likewise, an artificial neural network has been created for each area to estimate the percentage of free spaces, the output of which is ultimately the aforementioned sought-after variable.

As a result, the modelling for our problem is a two-level integrated model, the details of 
which are defined below:

\subsection{First Artificial Neural Network (ANN)}

The first artificial neural network is in charge of estimating the total number of tickets in the next time interval for a specific parking meter. Therefore, the response from this ANN will be the daily total of tickets sold up to a certain time.

We have opted to make this ANN a time series, given the relationship with time that the total number of tickets sold has by definition.

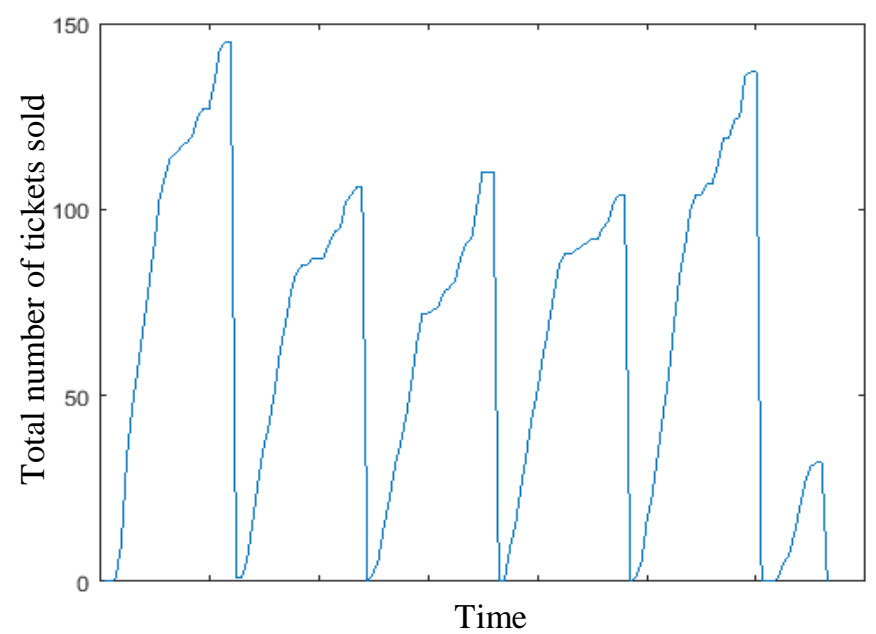

Figure 3.1 represents the total number of tickets sold during one week (Mon.-Sat.) in the month of February. As can be seen, more tickets are sold on Monday to Friday than on Saturday, since the use of the regulated area decreases due to work factors and also to a more reduced regulation time (only in the morning).

\section{Fig. 3.1 - Example of total tickets}

This behaviour appears in other time periods, such as the summer season, where the number of tickets sold decreases considerably.

In light of the analysis of the observed behaviour, we have considered other explanatory variables to be: time, day of the week, and the month, all of which are treated categorically.

\subsection{Second Artificial Neural Network (ANN)}

This ANN is in charge of estimating the percentage of free spaces in an area at a particular time; the estimated variable corresponds to the percentage expressed as a decimal.

The x-axis of Figure 3.2 represents the number of tickets sold in the last hour for each point of sale (parking meter), which represents a count. The y-axis represents the percentage of free spaces that each count contains. This way, it can be seen that the "number of tickets sold in the last hour" variable significantly explains the percentage of free spaces for the area in question.

However, it can be seen that this variable does not completely explain the percentage of free spaces; in the worst-case scenario (approximately 0.7 on the $\mathrm{x}$-axis), the variable to predict varies between approximately 0 to 0.65 , which is why more explanatory (input) variables are expected to be needed to make a better prediction. 


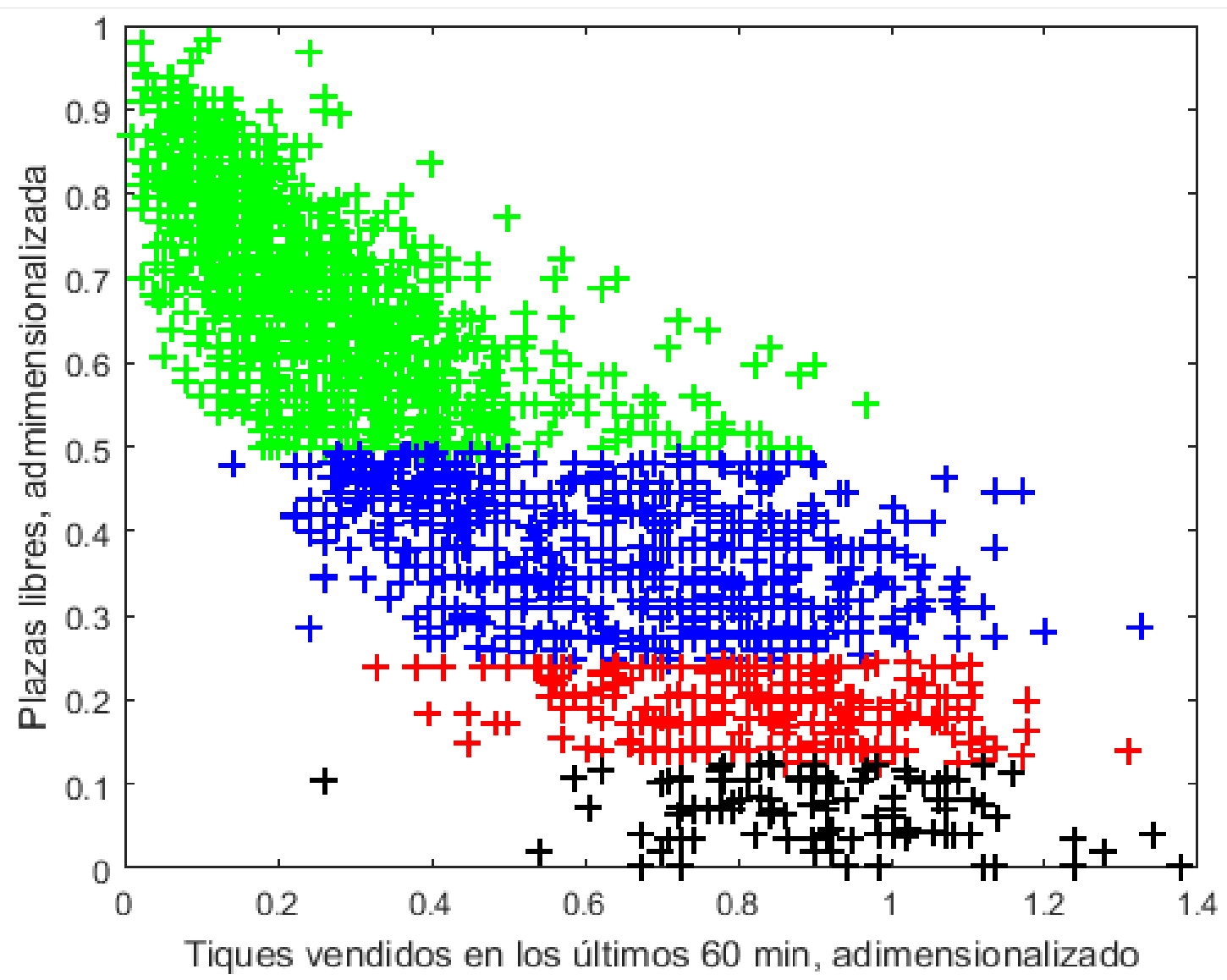

Fig. 3.2-Ratio of free spaces to tickets sold

To further refine the prediction, we have added three additional variables related to each of the following states: spaces occupied by residents, unavailable spaces, and spaces improperly occupied. These three have been chosen because they are independent of the previously described variable "tickets sold", thus adding important significance.

Also included are categorical time variables such as time, day of the week, and month. Finally, the categorical variable "weather" has been taken into account, which is activated at a particular threshold of daily precipitation.

\section{RESULTS}

\subsection{First Artificial Neural Network (ANN)}

A simple model consisting of 10 neurons and 2 delays has made it possible to obtain a relatively high correlation coefficient, an average $\mathrm{R}=0.995$ among all parking meters (approx. 200) without overfitting. The figure below (Fig. 4.1) shows the response achieved after training the model. 

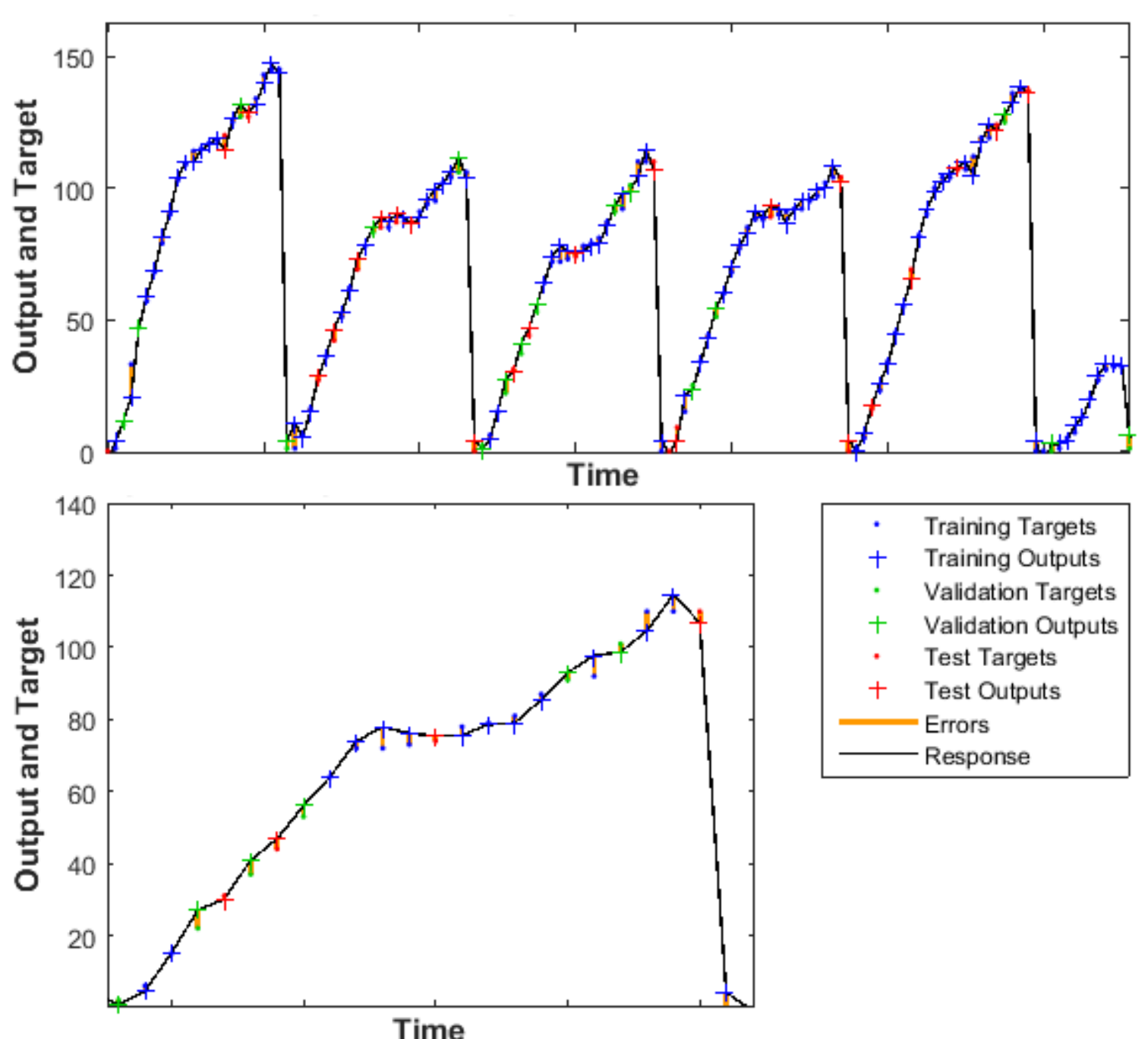

\section{Fig. 4.1 - Response example from ANN1}

\subsection{Second Artificial Neural Network (ANN)}

In this case, a model with 10 neurons allows good results to be obtained without overfitting. The training data are nothing more than the counts that have been made over the last year in the area in question, always considering the data deemed most fit after removing inconsistent data using a preliminary filtering algorithm for elimination before training.

Subsequent training of the network with cleaned data provides the results shown in Fig. 4.2. 


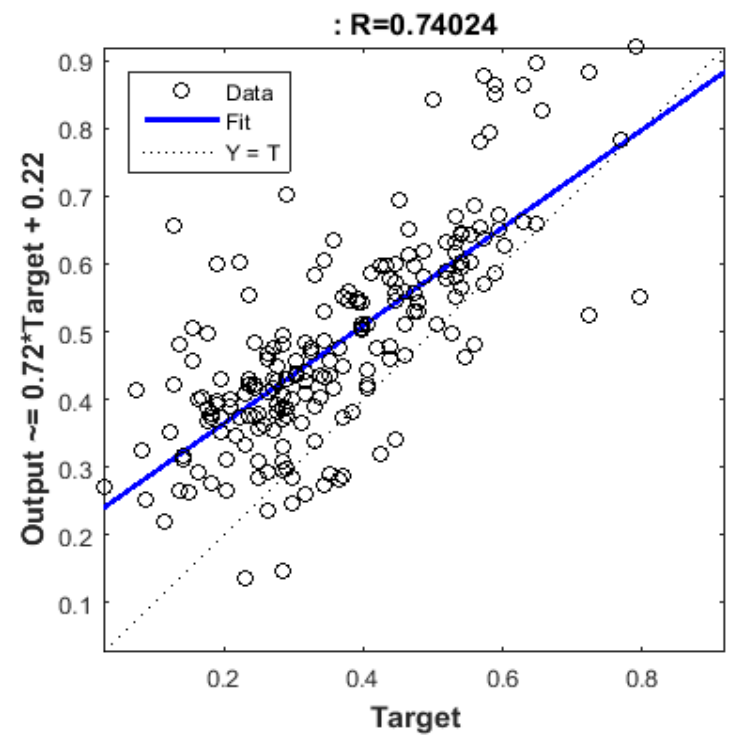

Fig. 4.2 - Comparison of ANN with the pilot

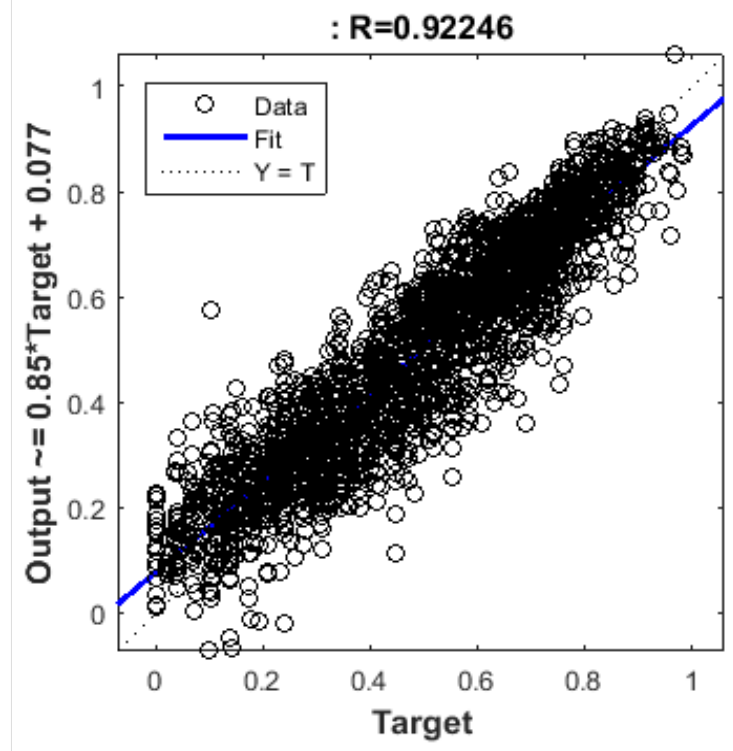

Fig. 4.3 - Training ANN2 for an area

The reliability of the predictions has been confirmed by a pilot count in a certain area for two weeks every half hour during the regulation time frame for the on-street parking. The results obtained are gathered in Fig. 4.3, which compares free spaces resulting from the pilot count with those predicted by the ANN.

\section{CONCLUSIONS}

The available data (both existing historical data and data obtained in real time) have made it possible to make a predictive model of the percentage of free spaces in each area.

In light of the results, a specialised software package has been developed that performs real- 
time estimates of the percentage of free spaces in each of the regulated areas into which the city of Seville is divided for each time interval. This application has a user interface where the results are published via smartphone app so that they can be consulted by users.

\section{REFERENCES}

National Oceanic and Atmospheric Administration. (2016). National Centers for Environmental Information. Retrieved from Climate Data Online: http://www.ncdc.noaa.gov/cdo-web/ 